\title{
A NEW KIND OF THE VARIANT OF THE MODIFIED BERNSTEIN-KANTOROVICH OPERATORS DEFINED BY "OZARSLAN AND DUMAN
}

\author{
Abhishek Kumar ${ }^{1}$ \\ ${ }^{1}$ IIT Roorkee
}

December 7, 2021

\begin{abstract}
In the present article, we dene a new kind of the modified Bernstein-Kantorovich operators defined by " Ozarslan (https://doi.org/10.1080/01630563.2015.1079219) i.e. we introduce a new function $\varsigma(\mathrm{x})$ in the modified Bernstein-Kantorovich operators defined by Ozarslan with the property $(\{)$ is an infinitely differentiable function on $[0 ; 1] ; \varsigma(0)=0 ; \varsigma(1)=1$ and $\varsigma^{\prime}(\mathrm{x})>0$ for all $\mathrm{x}[?][0 ; 1]$. We substantiate an approximation theorem by using of the Bohman-Korovkins type theorem and scrutinize the rate of convergence with the aid of modulus of continuity, Lipschitz type functions for the our operators and the rate of convergence of functions by means of derivatives of bounded variation are also studied. We study an approximation theorem with the help of Bohman-Korovkins type theorem in A-Statistical convergence. Lastly, by means of a numerical example, we illustrate the convergence of these operators to certain functions through graphs with the help of MATHEMATICA and show that a careful choice of the function $\varsigma(\mathrm{x})$ leads to a better approximation results as compared to the modified Bernstein-Kantorovich operators defined by Ozarslan (https://doi.org/10.1080/01630563.2015.1079219).
\end{abstract}

\section{Hosted file}

Paper_MMAS.pdf available at https://authorea.com/users/450002/articles/548447-a-new-kindof-the-variant-of-the-modified-bernstein-kantorovich-operators-defined-by-ozarslan-andduman 

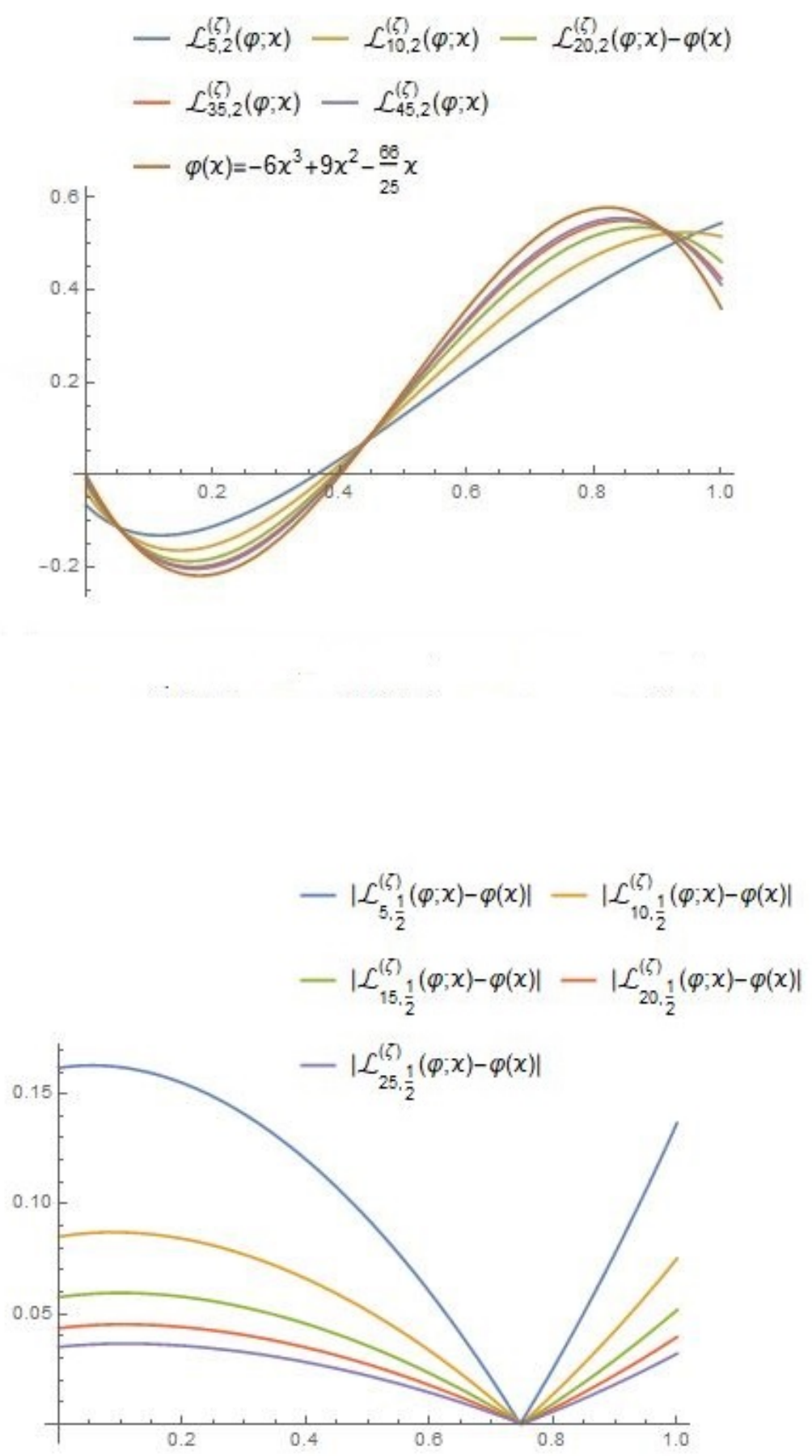


$$
\begin{aligned}
& -K_{10,1}(\varphi ; x)-\mathcal{L}_{10,1}^{(\zeta)}(\varphi ; x) \\
& -\varphi(x)=\chi^{4}-\chi^{3}-x+1
\end{aligned}
$$


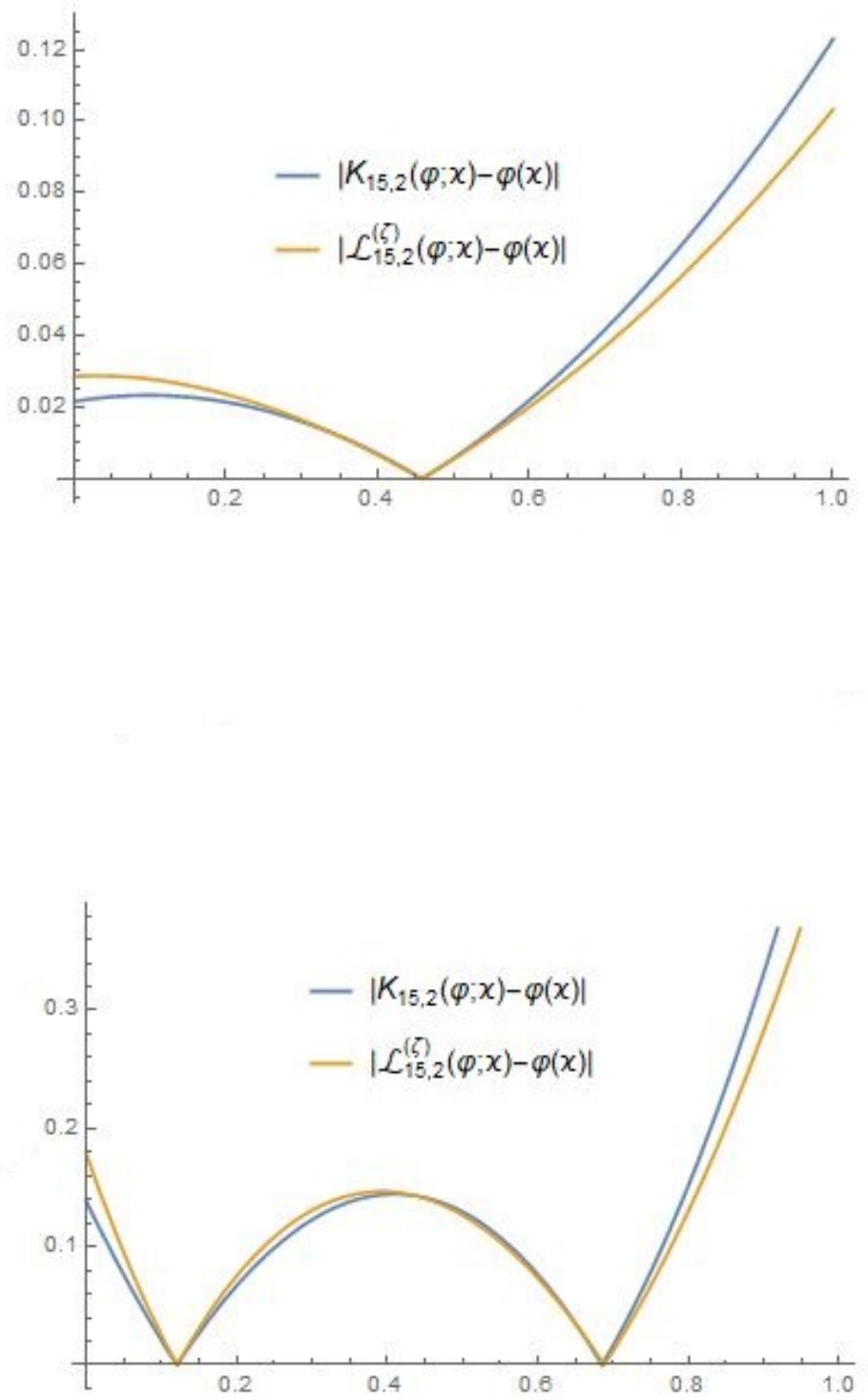